\title{
CMTX Disorder and CamKinase
}

\author{
Frédéric Bihel ${ }^{1}$, Burkhard Gess ${ }^{2,3}$ and Michel Fontés ${ }^{4 *}$ \\ 'Laboratoire d'Innovation Thérapeutique, Faculté de Pharmacie, UMR7200, Centre National de la Recherche Scientifique, \\ Université de Strasbourg, Strasbourg, France, ${ }^{2}$ Department of Sleep Medicine and Neuromuscular Disorders, University \\ Hospital Muenster, Muenster, Germany, ${ }^{3}$ Department of Neurology, Aachen RWTH University Clinic, Aachen, Germany, \\ ${ }^{4}$ Nutrition, Obesity and Risk of Thrombosis Laboratory, UMR Institut National de la Santé et de la Recherche Médicale 1062, \\ UMR INRA 1260, Aix Marseille Université, Marseille, France
}

Keywords: CamKinase, myelin sheath, neuropathies, genomic instability, drug development

Charcot-Marie-Tooth disease is a demyelinating peripheral neuropathy and is a heterogeneous inherited disorder (40 loci have been described so far) affecting peripheral nerves. Two forms, CMT1A and CMTX, account for 60 and 15\% of patients, respectively, with clear familial transmission (Boerkel et al., 2002).

CMTX is caused by mutations in the GBJ1 gene encoding the synthesis of connexin 32 (Cx32, Bergoffen et al., 1993), which is a myelin protein related to PNS and CNS (Scherer et al., 1995). $\mathrm{Cx} 32$ is a membrane protein located in gap junctions, which forms hexameric hemichannels called connexons. However, the mechanism by which anomalies in connexin 32 affect myelination and function of PNS remains is still unclear. Here, we share our opinion that CamKinase are involved in the pathophysiology of CMTX and likely involve genomic instability caused by mutations in Gjb1.

\section{CONNEXIN, CAMKII, AND GENOMIC STABILITY}

Apart from its role in the synthesis of $\mathrm{Cx} 32$, an inappropriate expression of the gene Gjb1 has recently been associated with genomic instability during mitosis (Mones et al., 2012, www.mitocheck.org) leading to the hypothesis that connexins may be linked, directly or indirectly, to genomic stability. The literature suggests that at least one signaling pathway is associated with both phenomena-calcium homeostasis. Indeed, connexin 32 oligomerizes in Schwann cells to form hemichannels known to facilitate the diffusion of metabolites across the layers of myelin sheath. It is now established that calmodulin $(\mathrm{CaM})$ can directly bind the C-terminus of $\mathrm{Cx} 32$ and regulate gap junction channels (Torok et al., 1997; Ahmad et al., 2001; Dodd et al., 2008; Stauch et al., 2012). In response to increased intracellular levels of calcium, CaM then binds to Cx32 channels triggering its closure and allowing the extrusion of $\mathrm{Ca}^{2+}$ via others channels to maintain calcium homeostasis. (Lurtz et al., 2003) In CMTX, anomalies of Cx32 alter the binding of CaM, leading to disturbance of calcium homeostasis by maintaining high intracellular calcium concentrations. (De Vuyst et al., 2006) However, as Cx32 anomalies decreases the binding of CaM, we can hypothesize an increase of "free" CaM in the presence of high concentrations of calcium leading to autophosphorylation of CaMKII upon activation by $\mathrm{Ca}^{2+}$ and $\mathrm{CaM}$ (Hell Johannes, 2014). Acting via a retrocontrol mechanism, CaMKII can then phosphorylate Cx32, leading to modulations of the gap junctional communications (Lampe and Lau, 2004; Stauch et al., 2012). Recently, we have shown that KN93 and KN-62-two inhibitors of reference of CaMKII-could restore connexin activity (Mones et al., 2014, p. 382), but as the level of phosphorylation of Cx32 has not been measured, we cannot make any conclusions about the mechanism involved in this overstimulation.

Genomic instability occurs when multipolar spindles appear due to centrosome overduplication. In 2002, Matsumoto and Maller described in Xenopus egg extracts that inactivation of CaMKII could block centrosome overduplication suggesting that calcium oscillations in the cell may be linked to centrosome duplication. (Matsumoto and Maller, 2002) Also also Xenopus egg extracts,

Citation: Bihel F, Gess B and Fontés M (2016) CMTX Disorder and CamKinase.

Front. Cell. Neurosci. 10:49. doi: 10.3389/fncel.2016.00049 
Reber et al. confirmed this hypothesis and showed that calcium or constitutive active CaMKII promotes microtubule destabilization (Reber et al., 2008). We recently showed that CamKII activity is overactivated in cells from a mouse model of CMTX involving mutations in Gjb1 (Mones et al., 2014) and in cells from CMTX1 patients (Mones et al., 2015). These cells presented centrosome overduplication and genomic instability. Interestingly, this cellular phenotype was corrected by treatment with CamKII inhibitors both in Gjb1-mutated mouse and human patient cells (Mones et al., 2014, 2015).

These data suggest that anomalies of Cx32 due to GBJ1 mutations lead to unbalanced calcium homeostasis resulting in a genomic instability through the overactivation of CaMKII.

\section{CONNEXIN AND MYELIN}

Myelin deficit is the first alteration observed in Cx32-deficient mice, supporting the link between connexin and myelin (Scherer et al., 1998). Cx32 is the most abundant connexin isoform in Schwann cells, and it oligomerizes to form gap junction channels between two cells. However, gap junctions in Schwann cells can also form intracellular channels through the layers of myelin providing a direct route for the diffusion of metabolites and second messengers from the Schwann cell to the axon. This is likely necessary for myelin formation (Ressot and Bruzzone, 2000). Our recent work with KN93 and KN62, two inhibitors of CaMKII, concur with this hypothesis because they may block the CaMKII-mediated phosphorylation of Cx32 and at least partially restore the activity of the connexon (Mones et al., 2014, 2015).

However, some facts challenge this mechanistic hypothesis. Indeed, Cx32 is the major component of liver gap junctions, and CMTX mutations of GJB1 do not result in a severe phenotype in this organ. Regarding CNS, although patients with central clinical signs have been reported (Hanemann et al., 2003), the majority of CMTX patients did not present central clinical signs. However, they did present infra-clinical and asymptomatic signs. This a major observation in inherited disorders caused by mutations in an ubiquitously expressed protein. There is usually no clear explanation.

\section{CAMKII AND MYELIN}

Another hypothesis to explain the CMTX phenotype due to GJB1 mutations is overactivation of CaMKII. A recent work by Weggener et al. supports this hypothesis. They showed that a mouse line invalidated for CamKII $\beta$ gene exhibits a deficit in myelination in the CNS. The authors suggested that this deficit is due to anomalies in phosphorylation of cytoskeletal proteins (Waggener et al., 2013). Earlier, Sahenk et al. reported that CMTX xenografts where Schwann cells bear mutations on Cx32 were presenting major cytoskeletal alterations (Sahenk, 1999). While the link between CaMKII, cytoskeleton anomalies and myelin deficits may be established, we do not yet know whether the CaMKII-related genomic instability observed in cell phenotypes of CMTX patients is part of the same signaling pathway leading to myelin deficit. However, this hypothesis is interesting because
CMTX-induced genomic instability in the Schwann cells may affect the PNS myelination in a downstream process occurring over a long period of time before the appearance of clinical symptoms. In another form of CMT (CMT1A), infants were showed to bear the CMT1A mutation, but without clinical symptoms. They still had a reduced nerve conduction velocity indicating that a deficit in myelination is already present before the disease manifests (Garcia and Combarros, 1998).

This begs the question of calcium homeostasis and myelination. Nobbio et al. demonstrated the impact of perturbation of calcium homeostasis in a CMT1A model (Nobbio et al., 2009). This is strengthened by the report of Stevens et al. demonstrating that perturbation of calcium homeostasis inhibits differentiation of precursors of Schwann cells (Stevens and Fields, 2000). In the same report, they suggested that it involves $\mathrm{Ca}^{2+}$, calmodulin and calmodulin kinase.

\section{DEMYELINATION AND INFLAMMATION}

Several mechanisms are likely co-existing between the primary causes of the disease (including gene mutation and the resulting deregulation of the downstream cascade) and the symptomatic manifestations of the disease (locomotion, fatigue, pain, etc.) In this field, Martini et al. demonstrated that murine models of demyelinating CMT present inflammation, in which both innate and adaptive immune systems would act as amplifiers of the primarily genetically caused neuropathies (for review, Martini and Willison, 2016). However, in their putative mechanism, they argued propose a yet to be identified primary activator that would lead to myelin phagocytosis by macrophages and Schwann cell dedifferentiation. The inflammatory process would be an aggravating factor of the neuropathy. Considering that inflammation is very frequently associated with degenerative disorders (Alzheimer's disease, diabetes ...), it is likely that inflammation acts in clinical picture of demyelinating CMT. The question of cause or consequence of the disorder is difficult to answer. However, the assumption that anomalies in tissues structure (abnormal myelin, decompacted myelin, etc), are leading to inflammation is very likely.

Our opinion is that inherited demyelinating disorders are developmental disorders linked to anomalies of Schwann cells terminal differentiation and myelin formation. However, this putative role of inappropriate differentiation as the primary cause of inflammation is still to be proved. In addition, potential direct links between genomic instability and neuroinflammation remain still elusive, although Taupin reported an association between neuroinflammation in Alzheimer disease and genomic instability (aneuploidy) (Taupin, 2010). We did not find a report of a similar phenomenon in PNS disorders.

\section{CMTX AND THERAPEUTIC APPROACHES}

Therapeutic efficacy requires a good understanding of the mechanisms involved in the disease. Recent works in the CMTX field show that there are many questions still pending before this 
genetic disease can be fully understood. Inflammation is certainly a good target that could lower disease severity including pain. Targeting upstream events linked to the primary cause of the disorder would be also a promising track to treat CMTX patients. Indeed, we have demonstrated that in vitro and in vivo abnormal phenotypes are corrected in animal models by treatment with CamKII inhibitors (Mones et al., 2014). In addition, we have demonstrated that the same anomalies are observed in cells from CMTX patients and that these anomalies are corrected using CamKII inhibitors. CaMKII is a common target to the various mechanistic hypotheses presented earlier. In order to prevent potential toxicity, development of CaMKII modulators may allow down-activation of CaMKII in the PNS without strongly altering calcium homeostasis in other cells. The CamKII inhibitors KN93 and KN62 have never been tested in humans and deserve a full optimization process to improve their efficacy toward CamKII. However, although no toxic evaluation has been reported, no adverse effect has been reported in animals treated with this molecule.

The hypothesis dealing with genomic instability and myelin deficit is also interesting in terms of therapeutic options. Indeed, several kinases are already known to be involved in genomic instability. These likely act downstream to CamKinases.

\section{REFERENCES}

Ahmad, S., Martin, P. E., and Evans, W. H. (2001). Assembly of gap junction channels: mechanism, effects of calmodulin antagonists and identification of connexin oligomerization determinants. Eur. J. Biochem. 268, 4544-4552. doi: 10.1046/j.1432-1327.2001.02380.x

Bergoffen, J., Scherer, S. S., Wang, S., Scott, M. O., and Bone, L. J. (1993). Connexin mutations in X-linked Charcot-Marie-Tooth disease. Science 262, 2039-2042. doi: $10.1126 /$ science. 8266101

Boerkel, C. F., Takashima, H., Garcia, C. A., Olney, R. K., Johnson, J., and Berry, K. (2002). Charcot-Marie-Tooth disease and related neuropathies: mutation distribution and genotype-phenotype correlation. Ann. Neurol. 51, 190-201. doi: 10.1002/ana.10089

De Vuyst, E., Decrock, E., Cabooter, L., Dubyak, G. R., Naus, C. C., Evans, W. H., et al. (2006). Intracellular calcium changes trigger connexin 32 hemichannel opening. EMBO J. 25, 34-44. doi: 10.1038/sj.emboj.7600908

Dodd, R., Peracchia, C., Stolady, D., and Török, K. (2008). Calmodulin association with connexin32-derived peptides suggests trans-domain interaction in chemical gating of gap junction channels. J. Biol. Chem. 283, 26911-26920. doi: 10.1074/jbc.M801434200

Garcia, A., and Combarros, A. (1998). Charcot-Marie-Tooth disease type $1 \mathrm{~A}$ with $17 \mathrm{p}$ duplication in infancy and early childhood: a longitudinal clinical and electrophysiologic study. Neurology 50, 1061-1067. doi: 10.1212/WNL.50.4.1061

Hanemann, C. O., Bergmann, C., Senderek, J., Zerres, K., and Sperfeld, A. D. (2003). Transient, recurrent, white matter lesions in X-linked Charcot-MarieTooth disease with novel connexin 32 mutation. Arch. Neurol. 60, 605-609. doi: 10.1001/archneur.60.4.605

Hell Johannes, W. (2014). CaMKII: claiming center stage in postsynaptic function and organization. Neuron 81, 249-265. doi: 10.1016/j.neuron.2013.12.024

Lampe, P. D., and Lau, A. F. (2004). The effects of connexin phosphorylation on gap junctional communication. Internat. J. Biochem. Cell Biol. 36, 1171-1186. doi: 10.1016/S1357-2725(03)00264-4

Lurtz, M. M., and Louis, C. F. (2003). Calmodulin and protein kinase C regulate gap junctional coupling in lens epithelial cells. Am. J. Physiol. Cell Physiol. 285, C1475-C1482. doi: 10.1152/ajpcell.00361.2002
Pim1 is one of these kinases that is involved in genomic instability in cancer cells (Roh et al., 2003). We have shown that Pim1 inhibitors can correct genomic instability in GBJ1mutated cell lines, but were unable to correct Cx32 activity (Mones et al., 2014). It would be interesting to test these Pim1 inhibitors in CMTX mice to evaluate their ability to restore a normal phenotype, and validate or rule out this mechanistic hypothesis. Moreover, downstream kinases could be an additional therapeutic target.

\section{AUTHOR CONTRIBUTIONS}

FB and MF wrote the manuscript. BG critically reviewed the manuscript. All authors read and approved the final version of the manuscript.

\section{ACKNOWLEDGMENTS}

This article is dedicated to S. Mones who published most of the cited papers. Saleh was a MD and defended his $\mathrm{PhD}$ thesis in 2014. He returns now to Syria, and was incorporated in Syrian army as military doctor. He was killed Saturday September 10th in a suicide terrorist attack of his hospital.

Martini, R., and Willison, H. (2016). Neuroinflammation in the peripheral nerve: cause, modulator, or bystander in peripheral neuropathies? Glia 64, 475-486. doi: 10.1002/glia.22899

Matsumoto, Y., and Maller, J. L. (2002). Calcium, calmodulin, and CaMKII requirement for initiation of centrosome duplication in Xenopus egg extracts. Science 295, 499-502. doi: 10.1126/science.1065693

Mones, S., Bordignon, B., and Fontés, M. (2012). Connexin 32 is involved in mitosis. Glia 60, 457-464. doi: 10.1002/glia.22279

Mones, S., Bordignon, B., Peiretti, F., Landrier, J. F., Gess, B., Bourguignon, J. J., et al. (2014). CamKII inhibitors reduce mitotic instability, connexon anomalies and progression of the in vivo behavioral phenotype in transgenic animals expressing a mutated Gjb1 gene. Front. Neurosci. 8:151. doi: 10.3389/fnins.2014.00151

Mones, S., Gess, B., Bordignon, B., Altié, A., Young, P., Bihel, F., et al. (2015). CMTX1 patients' cells present genomic instability corrected by CamKII inhibitors. Orph. J. Rare Dis. 10, 56. doi: 10.1186/s13023-0150270-5

Nobbio, L., Sturla, L., Fiorese, F., Usai, C., Basile, G., Moreschi, I., et al. (2009). P2X7-mediated increased intracellular calcium causes functional derangement in schwann cells from rats with CMT1A neuropathy. J. Biol. Chem. 284, 23146-23158. doi: 10.1074/jbc.M109.027128

Reber, S., Over, S., Kronja, I., Gruss, O. J. (2008). CaM kinase II initiates meiotic spindle depolymerization independently of APC/C activation. CaM kinase II initiates meiotic spindle depolymerization independently of APC/C activation. J. Cell Biol. 183, 1007-1017. doi: 10.1083/jcb.2008 07006

Ressot, C., and Bruzzone, R. (2000). Connexin channels in Schwann cells and the development of the X-linked form of Charcot-Marie-Tooth disease. Brain Res. Rev. 32, 192-202. doi: 10.1016/S0165-0173(99)00081-8

Roh, M., Gary, B., Song, C., Said-Al-Naief, N., Tousson, A., Kraft, A., et al. (2003). Overexpression of the oncogenic kinase Pim-1 leads to genomic instability. Cancer Res. 63, 8079-8084.

Sahenk, Z. (1999). "Abnormal Schwann cell-axon interactions in CMT neuropathies - the effects of mutant Schwann cells on the axonal cytoskeleton and regeneration-associated myelination," in Charcot-MarieTooth Disorders, eds M. E. Shy, J. Kamholz, and R. E. Lovelace (New York, 
NY: New York Acad Sciences), 415-426. doi: 10.1111/j.1749-6632.1999.tb 08602.X

Scherer, S. S., Deschênes, S., Xu, Y. T., Grinspan, J. B., Fischbeck, K. H., and Paul, D. L. (1995). Connexin 32 is a myelin-related protein in the PNS and CNS. J. Neurosci. 15, 8281-8194.

Scherer, S. S., Xu, Y.-T., Nelles, E., Fischbeck, K., Willecke, K., and Bone, L. J. (1998). Connexin32-null mice develop demyelinating peripheral neuropathy. Glia 24, 8-20. doi: 10.1002/(SICI)1098-1136(199809)24:1<8::AID-GLIA2>3. $0 . \mathrm{CO} ; 2-3$

Stauch, K., Kieken, F., and Sorgen, P. (2012). Characterization of the structure and intermolecular interactions between the Connexin 32 carboxylterminal domain and the protein partners synapse-associated protein 97 and calmodulin. J. Biol. Chem. 287, 27771-27788. doi: 10.1074/jbc.M112.382572

Stevens, B., and Fields, R. D. (2000). Response of schwann cells to action potentials in development. Science 287, 2267-2271. doi: 10.1126/science.287.5461.2267

Taupin, P. (2010). Aging and neurogenesis, a lesion from Alzheimer's disease. Aging Dis. 1, 158-168.
Torok, K., Stauffer, K., and Evans, W. H. (1997). Connexin 32 of gap junctions contains two cytoplasmic calmodulin-binding domains. Biochem. J. 326, 479-483. doi: 10.1042/bj3260479

Waggener, C. T., Dupree, J. L., Elgersma, Y., and Fuss, B. (2013). CaMKII $\beta$ regulates oligodendrocyte maturation and CNS myelination. J. Neurosci. 33, 10453-10458. doi: 10.1523/JNEUROSCI.5875-12.2013

Conflict of Interest Statement: The authors declare that the research was conducted in the absence of any commercial or financial relationships that could be construed as a potential conflict of interest.

Copyright (c) 2016 Bihel, Gess and Fontés. This is an open-access article distributed under the terms of the Creative Commons Attribution License (CC BY). The use, distribution or reproduction in other forums is permitted, provided the original author(s) or licensor are credited and that the original publication in this journal is cited, in accordance with accepted academic practice. No use, distribution or reproduction is permitted which does not comply with these terms. 